\title{
Simulating a Wavelength-Size 2-D Lens with an Accurate Numerical Method
}

\author{
Artem V. Boriskin, Alexander I. Nosich, Ayhan Altintas* \\ Institute of Radiophysics and Electronics \\ of the National Academy of Sciences of Ukraine, Kharkov 61085, Ukraine \\ * Bilkent University,06533 Ankara, Turkey \\ e-mail: a_boriskin@yahoo.com
}

\section{ABSTRACT}

The effect of a localized light source directivity improvement due to an arbitrarily shaped dielectric cylinder taken as a 2-D model of a dielectric lens is studied. The source is simulated by the field of a Complex SourcePoint (CSP). An efficient algorithm for the solution of 2D problem of wave scattering by a smooth dielectric cylinder is developed, based on the concept of analytical regularization. Basic properties of the algorithm are studied. Numerical results for the accuracy of the algorithm and sample far-field characteristics such as total radiated power, directivity and radiation patterns for various lens parameters are presented.

\section{INTRODUCTION}

In the optical and microwave ranges, dielectric lenses and rods are frequently used to focus the radiation or improve its directive character. However, analysis and design of lenses frequently involves a number of highfrequency approximations neglecting the lens edges and not taking a full account of all the interactions between different parts of the scattering objects [1]. If done numerically, it is often based on the discretizations having unclear and uncontrollable accuracy. Another commonly faced drawback which may spoil the wavelength-scale lens analysis is due to modeling of the incident field as a dipole field [2] or a Gaussian beam [3]. This is because the former field has a fixed shape, and the latter does not satisfy the Helmholtz equation. Therefore, there is a need in developing a more adequate simulation technique able to serve as a promising CAD tool.

\section{MATHEMATICAL MODEL}

The 2-D model of a dielectric lens scattering is discussed, as shown in Fig.1.

Lens: The scatterer is taken as a homogeneous isotropic dielectric cylinder with a so-called "super-elliptic" crosssection [4] that simulates a rectangle with rounded edges. The curve $L$ bounding the lens cross-section is determined by the following equation:

$$
[x / l a]^{2 v}+[y / a]^{2 v}=1, \quad 0<v<\infty
$$

where $l$ is the axial ratio and parameter $v$ determines the

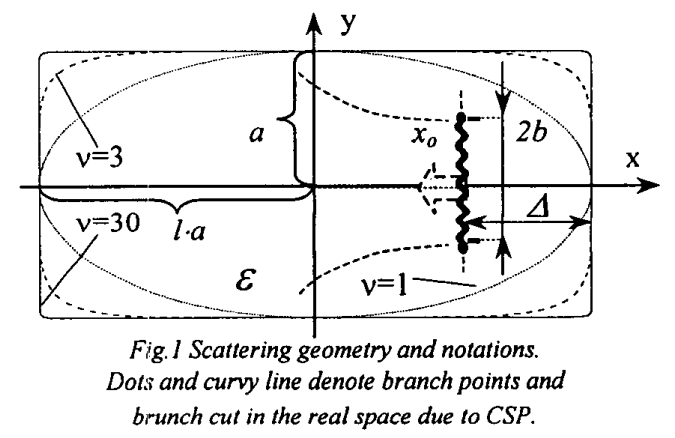
supper-ellipse corner rounding. The greater the value of the parameter $v$, the closer the cross-section contour to a rectangle ( $v=1$ corresponds to an ellipse), however for all $v>1 / 2$ it is a twice-differentiable curve.

Source: The incident field is generated by a CSP line source located at $\mathbf{r}_{c s}$. The main advantages of the CSP field

$$
U_{0}(\mathbf{r})=H_{0}^{(1)}\left(k\left|\mathbf{r}-\mathbf{r}_{c s}\right|\right)
$$

are that it is an exact solution of the Helmholtz equation with respect to the observation point, and has a variable beam width controlled by parameter $k b[5]$. Here $k$ is the free-space wavenumber, and the complex source position vector is

$$
\mathbf{r}_{c s}=\left\{x_{c s}, y_{c s}\right\}=\mathbf{r}_{0}+i \mathbf{b}, \mathbf{r}_{0}=\left\{x_{0}, y_{0}\right\}, \mathbf{b}=\{b \operatorname{Cos} \beta, b \operatorname{Sin} \beta\}
$$

This work was supported by The IEEE MTT Society and by The TUBITAK (Scientific and Technical Research Council of Turkey) in the form of fellowships to the first author. The aid of Dr. S.V. Boriskina in developing the computer code is acknowledged with gratitude. 
Parameters $b$ and $\beta$ can be associated with the width and orientation angle of a light-emitting aperture simulated by a CSP. Note that the CSP excitation depicted in Fig. 1 imply that $\beta=\pi$. Hence, the branch points of $U_{0}$ in the real space are located at $\left(x_{0}, y_{0} \pm b\right)$, with $y_{0}=0$.

Basic IEs and analytical regularization: The total field has to satisfy the Helmholtz equation with the coefficients $k$ and $k_{e}=k \sqrt{\varepsilon}$ inside and outside the lens, respectively. After splitting out the incident CSP field, it is presented in terms of the single-layer potentials with the density functions to be determined:

$$
U^{r a d}(\mathbf{r})=\int \varphi\left(\mathbf{r}_{s}\right) G_{0}\left(\mathbf{r}, \mathbf{r}_{s}\right) d l_{s}, U^{s c}(\mathbf{r})=\int \psi\left(\mathbf{r}_{s}\right) G_{e}\left(\mathbf{r}, \mathbf{r}_{s}\right) d l_{s}
$$

where the kernels are the Green's functions of the free space and uniform media of relative permittivity $\mathcal{E}$, respectively:

$$
G_{0}\left(\mathbf{r}, \mathbf{r}_{s}\right)=\frac{i}{4} H_{0}\left(k\left|\mathbf{r}-\mathbf{r}_{s}\right|\right), G_{e}\left(\mathbf{r}, \mathbf{r}_{s}\right)=\frac{i}{4} H_{0}\left(k_{e}\left|\mathbf{r}-\mathbf{r}_{s}\right|\right)
$$

and $H_{m}$ is the Hankel function of the first kind

By applying the boundary conditions, a set of singular integral equations (IEs) is obtained. Extraction and analytical inversion of the singular parts of the IEs are done by adding and subtracting the canonical-shape operators with kernels $\stackrel{0}{G}_{0\{e\}}=\frac{i}{4} H_{0}\left(2 k_{\{e\}} a \operatorname{Sin}\left|\left(t-t_{s}\right) / 2\right|\right)$, and their normal derivatives, respectively, and by using a Galerkin scheme with angular exponents as global basis functions. This results in a coupled pair of infinite-matrix equations having favorable features:

$$
\left\{\begin{array}{l}
\varphi_{m} H_{m}(k a) J_{m}(k a)-\psi_{m} H_{m}\left(k_{e} a\right) J_{m}\left(k_{e} a\right)+\sum_{n=-\infty}^{\infty}\left[\varphi_{n} A_{n m}-\psi_{n} B_{n m}\right]=-u_{m} \\
\alpha \cdot k a \varphi_{m} H_{m}^{\prime}(k a) J_{m}(k a)-k_{e} a \psi_{m} H_{m}\left(k_{e} a\right) J_{m}^{\prime}\left(k_{e} a\right)+\sum_{n=-\infty}^{\infty}\left[\alpha \cdot \varphi_{n} C_{n m}-\psi_{n} D_{n m}\right]=-\tilde{u}_{m}
\end{array} \quad m=0, \pm 1, \pm 2, \ldots\right.
$$

where the matrix elements $A_{n m}, B_{n m}, C_{n m}, D_{n m}$ and $u_{m}, \tilde{u}_{m}$ are obtained as Fourier-expansion coefficients, with integrands given by the smooth functions. The latter are the differences between (5) on $L$ and on the canonical-shape contour, i.e. a circle of radius $a$. The coefficient $\alpha$ is 1 or $\varepsilon$ for $\mathrm{E}$ - or $\mathrm{H}$ - polarization, respectively. Equation (6) can be easily transformed to a classical block-type Fredholm second-kind matrix equation.

Such a regularization guarantees point-wise convergence of the numerical solution, i.e., a possibility to minimize the error to machine precision by solving progressively greater matrices. The rate of convergence of the algorithm can be estimated by plotting the normalized computational error, $e(N K)$, in the sense of the $l_{2}^{2}$ norm, versus the matrix truncation number $N K$ :

$$
e(N K)=\left(\sum_{|n| \leq N K}\left[\left|\varphi_{n}^{N K}-\varphi_{n}^{N K+1}\right|^{2}+\left|\psi_{n}^{N K}-\psi_{n}^{N K+1}\right|^{2}\right]\right)^{1 / 2} /\left(\sum_{|n| \leq N K}\left[\left|\varphi_{n}^{N K}\right|^{2}+\left|\psi_{n}^{N K}\right|^{2}\right]\right)^{1 / 2}
$$

where $\varphi_{n}^{N K}, \psi_{n}^{N K}$ are the expansion coefficients computed from the matrix equation with each block truncated after $N K$ equations. Details of the algorithm properties can be found in [6].

In application to dielectric scatterers, this approach is also free from the heavy inaccuracies near to natural resonances that are intrinsic for conventional numerical approximations [7].

Field characteristics: As the far-field characteristics are of interest, the large-r approximation is used. This enables one to replace the Hankel functions with its asymptotic and to reduce the first integral of (4) to

$$
U^{r a d}(t)=H_{0}(k r) \cdot \Phi(t)=\left[(1 / i \pi k r)^{1 / 2} \cdot e^{i k r}\right] \cdot \frac{1}{2 \pi} \sum_{n=-\infty}^{\infty} \varphi_{n} \int_{0}^{2 \pi} \exp \left[-i k\left(\operatorname{Cos}(t) x\left(t_{s}\right)+\operatorname{Sin}(t) y\left(t_{s}\right)\right)\right] \cdot e^{\mathrm{int} t_{s}} d t_{s}
$$

where $\Phi(t)$ is the far-field radiation pattern.

The total radiated power is found by integrating the Pointing vector radial component over a circle of large radius $r \rightarrow \infty$ and can be presented as a series in the following way: 


$$
P^{r a d}=\alpha_{0} \frac{2}{\pi} \int_{0}^{2 \pi}|\Phi(t)|^{2} d t=4 \alpha_{0}\left|\sum_{n=-\infty}^{\infty} \sum_{m=-\infty}^{\infty} \varphi_{n} Y_{m n}\right|^{2}
$$

where $Y_{m n}$ are the Fourier-expansion coefficients, with the integrand given by the corresponding factor in (8), and $\alpha_{0}$ is $Z_{0}$ or $Z_{0}^{-1}$, in the case of the E- and $\mathrm{H}$ - polarization, respectively, $Z_{0}=\left(\varepsilon_{0} / \mu_{0}\right)^{1 / 2}$ is the free space impedance.

Based on the series representation, main-beam directivity is determined as:

$$
D=\frac{\pi}{2}\left|\sum_{n=-\infty}^{\infty} \sum_{m=-\infty}^{\infty} \varphi_{n} \cdot Y_{m n} e^{i m t_{\max }}\right|^{2} /\left|\sum_{n=-\infty m=-\infty}^{\infty} \sum_{n}^{\infty} \varphi_{n} \cdot Y_{n ! n}\right|^{2}
$$

Note that numerical results for the total radiated power and directivity should be compared to the same values for a CSP located in free space:

$$
P_{0}=\alpha_{0} \frac{\pi}{2} I_{0}(2 k b), \quad D_{0}=2 \pi\left|\sum_{n=-\infty}^{\infty} J_{n}\left(k r_{c s}\right) \cdot e^{-i n \varphi_{\max }}\right|^{2} \cdot\left(\sum_{n=-\infty}^{\infty} \mid J_{n}\left(k r_{c s}\right)^{2}\right)^{-1}
$$

where $I_{0}$ is the modified Bessel function.

\section{NUMERICAL RESULTS}

One can see (Fig.2.) that the value of the relative error decreases with increasing of $N K$. However it is important to see what is the rate of that decrement and what is its dependence on the scatterer parameters. The analysis of the plots shows that accuracy guaranteed by choosing $N K \approx(k a \sqrt{\varepsilon}+1)(l+v)-k a+30 / k a$ is 2 digits if $k a<3$ and 3 digits if $k a>3$. The corresponding truncation number values are pointed with arrows and connected with dash-dotted line. In general, a check of the numerical error behavior should always precede the computations of the lens performance.

Figs. 3 and 4 enable one to optimize the lens directivity by varying the CSP distance from the back end of the rodlike lens and by changing the rod length, as controlled by parameters $k \Delta$ and $k a l$, respectively. One can see that the main maximum of $\mathrm{D}(k \Delta)$ is achieved with $k \Delta \approx 0.7$ that corresponds approximately to $\lambda_{e} / 8$, where $\lambda_{e}$ is the wavelength in dielectric. Increasing of the shift results in a fall of directivity. Note that here the wavelength is fixed and controlled by parameter $k a$.

Fig. 4 shows that the directivity as a function of the normalized length is also non-monotonic. If $k a=1$, it displays a series of maxima with the greatest at the length of the rod al equal approximately to $3 \lambda_{e}$. Note that the periodical sequences of extrema are explained by internal resonances. Their period is around $0.25 \lambda_{e}$.

Two curves in the figure correspond to the directivity in the direction of the maximum lobe of radiation pattern and along the symmetry plane given by $\beta=180^{\circ}$, respectively. The direction of the maximum lobe versus the normalized length is given in Fig. 5. The normalized radiated power as a function of the normalized rod length is given in Fig. 6. Finally, Fig. 7 presents sample far-field radiation patterns for different antenna sizes in the E- polarization. One can see that the effect of spatial compression of the originally almost omnidirectional radiation really takes place. Besides, the splitting of the main-lobe is also well seen that makes intuitive consideration "the longer rod, the better" invalid and calls for a full-wave analysis.

As a resonant nature of the effects is clearly seen a further research is required and will be done by the presented approach.

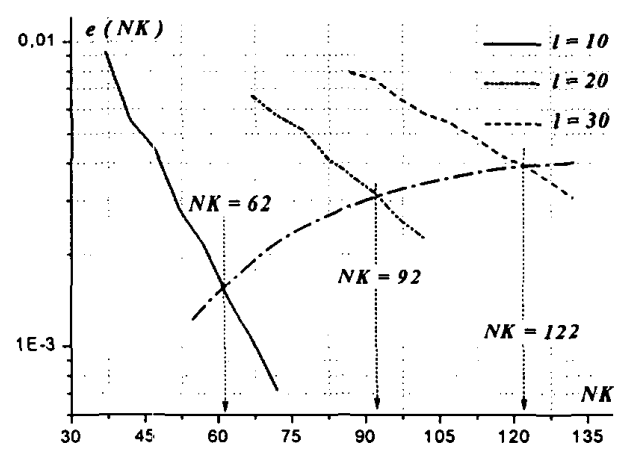

Fig. 2. Commutations error versus truncation number. $k a=1, \varepsilon=2.5+i 0.0, v=1$, $k b=0.1, \beta=180, k \Delta=0.7, y_{0} / a=0$.

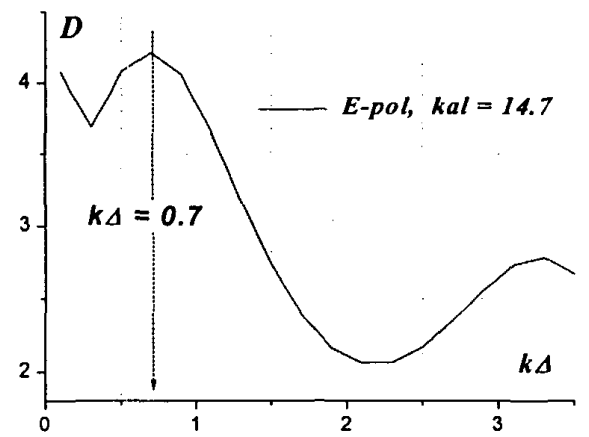

Fig. 3. Directivity versus normalized shift parameter $k \Delta=k a l-k x_{0}$ $k a=1, \varepsilon=2.5, v=1, k b=0.1, \beta=180, y_{0} / a=0$. 

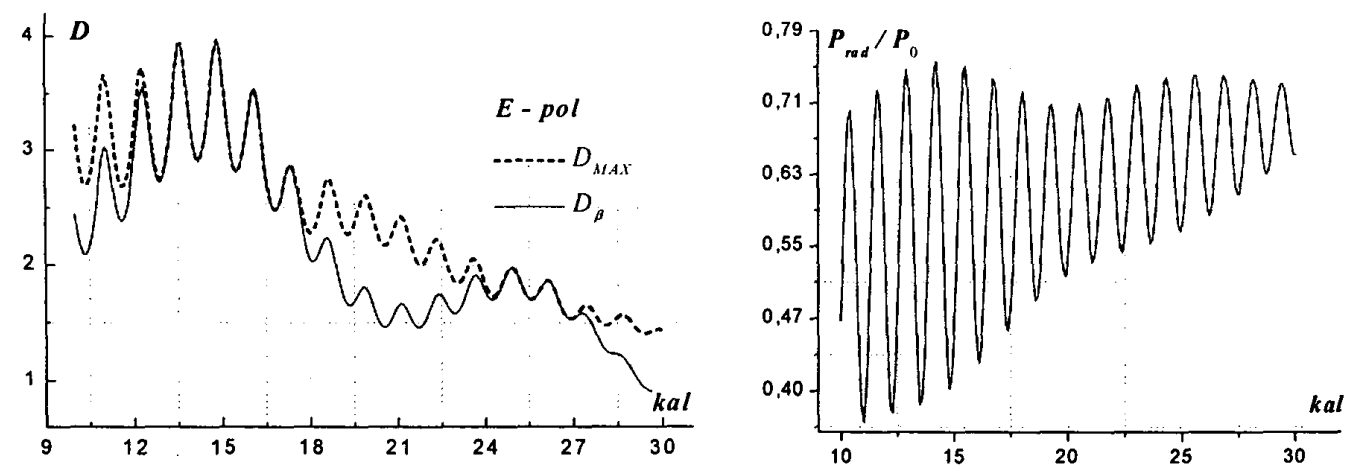

Fig. 4. Directivity versus the normalized rod-length parameter Dashed and solid lines are for Directivity in the maximal lobe and CSP axis direction, respectively.

$k a=1, \varepsilon=2.5, v=1, k b=0.1, \beta=180, k \Delta=0.7, y_{0}=0$.

The directivity of the CSP in the free space is $D_{0}=0.042$.

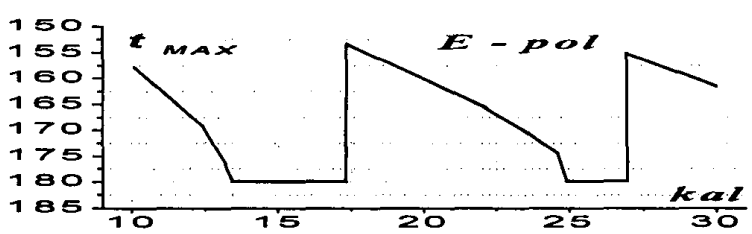

Fig. 5. The direction of the maximum lobe of the radiation patter versus the rod length (for the same parameters as in Fig.4)

\section{CONCLUSIONS}

An efficient and accurate numerical solution, based on the analytical regularization, to the $2 \mathrm{D}$ problem of the wave scattering by an arbitrary shaped smooth dielectric

Fig. 6. Radiated power normalized by the power of the CSP in the free space versus rod-length.

$k a=1, \varepsilon=2.5, v=1, k b=0.1, \beta=180, k \Delta=0.7, y_{0}=0$.

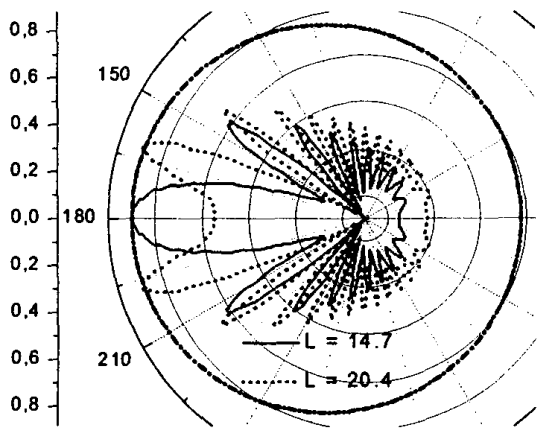

Fig. 7. Normalized radiation patterns corresponding to the maximum and minimum of the directivity in Fig. 4. Dashdotted line is for the pattern of the same CSP in the free space

$k a=1, \varepsilon=2.5, v=1, k b=0.1, \beta=180, k \Delta=0.7, y_{0}=0$. cylinder has been used to analyze the dielectric rod-like lens performance. Though, as it is shown, the algorithm can generate data with up to digital precision, a uniform 2-digit accuracy was kept for numerical results. It has been demonstrated that placing a light-emitting source inside a dielectric rod-like lens leads to a significant improvement of directivity. Also the importance of the right choice of CSP location with respect to the back end of the rod and the rod length has been shown. The given sample far-field radiation patterns demonstrate the effect of focusing if these parameters are chosen properly.

The presented approach can be easily modified to cover the case of a beam source located outside the dielectric lens. Such a geometry can be used to simulate an external laser-beam input to an optical lens or a section of optical fiber.

\section{REFERENCES}

[1] C. Salema, C. A. Fernandes, R.K. Jha, Solid Dielectric Horn Antennas, Boston: Artech House, 1998.

[2] H. Mieras, 'Radiation pattern computation of a spherical lens using Mie series', IEEE Trans. on Antennas Propagat., vol. AP-30, no. 6, pp. 1221-1224, 1982.

[3] E. E. M. Khaled, S. C. Hill, P. W. Barber, 'Scattered and internal intensity of a sphere illuminated with a Gaussian beam', IEEE Trans. Antennas Propagat., vol. 4, no. 3, pp. 1221-1224, 1999.

[4] M.Gardner, 'Mathematical games', Scientific American, vol. 213, no. 3, pp. 222-232, 1965.

[5] L. B. Felsen, 'Complex-point source solutions of the field equations and their relation to the propagation and scattering of the Gaussian beams', Symp. Mathem., vol. 18, pp. 39-56, 1975.

[6] S.V.Boriskina, A.I. Nosich, 'Fast and accurate in resonances solution to the scattering by arbitrary dielectric cylinders based on the canonical-shape inversion', IEEE Trans. Antennas Propagat., 2001, submitted.

[7] G.L. Hower, et al., 'Inaccuracies in numerical calculations of scattering near natural frequencies of penetrable objects', IEEE Trans. Antennas Propagat., vol. AP-41, no. 7, pp. 982-986, 1993. 